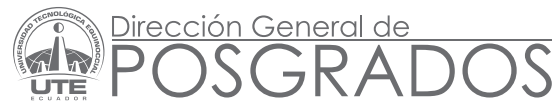 \\ EIDOS
}

\section{Cultura organizativa: análisis de su relación con la estrategia y gestión empresarial}

\author{
${ }^{1}$ Geovanni Basabe Moreno, ${ }^{2}$ Mauricio Basabe Moreno \\ ${ }_{1}^{1}$ Asociación de Municipalidades Ecuatorianas, AME, Quito - Ecuador, geovanni.basabe@ame.gob.ec \\ 2 Universidad Tecnológica Equinoccial, UTE, Quito - Ecuador, mauricio.basabe@ute.edu.ec
}

Recepción/Received: 30, 09, 2013

Aceptación/Accepted: 26, 11, 2013

Publicado/Published: 20, 12, 2013

\section{Resumen:}

A partir de la idea de que la cultura organizativa es un recurso empresarial clave para consolidar y preservar una ventaja competitiva sostenible en el tiempo, este artículo presenta un análisis sobre la manera como influye y condiciona el correcto funcionamiento de las empresas, orientado a lograr niveles óptimos de efectividad y eficiencia económica. De acuerdo con la estructura constitutiva del documento, se inicia reseñando la evolución y desarrollo de la Dirección Estratégica Empresarial para, luego de establecer una aproximación conceptual, determinar su influencia en la gestión empresarial con énfasis sobre la efectividad, dirección estratégica y su trato como recurso intangible.

\section{Palabras clave:}

Cultura organizativa, dirección estratégica, efectividad económica, ventaja competitiva sostenible, entorno económico, recurso intangible.

\section{Abstract:}

Since the idea than the organizational culture are a key business resource to consolidate and preserve a competitive sustainable advantage in the time, this article presents an analysis on the way how it influences and determines the correct functioning of the companies orientated to achieving ideal levels of effectiveness and economic efficiency. In agreement to the constitutive structure of the document, it begins outlining the evolution and development of the managerial strategic management for, after establishing a conceptual approximation, determining his influence in the business management emphatically on the effectiveness, strategic management and his treatment as intangible resource.

\section{Keywords:}

Organizational culture, strategic management, economic efficiency, competitive sustainable advantage, economic environment, intangible resource. 


\section{INTRODUCCIÓN}

Habitualmente la dirección estratégica de las empresas, con el propósito de poder identificar aquellos factores de mejora continua que pueden conducir a las organizaciones hacia una etapa superior respecto de la consecución de sus resultados, opta por determinados indicadores económicos y financieros, identificados a partir de variables de naturaleza cuantitativa, fácilmente medibles, que permiten adoptar aquellas medidas correctoras del caso, en base de desviaciones que se registran, por las actividades de control y de seguimiento concurrente o a posteriori que se ejecutan.

Pero esta actividad imperativa de monitoreo -por lo general-, descuida un aspecto que se reviste de particular importancia, que posee una dimensión etérea y es complejo de cuantificar, como lo es la "cultura organizativa". Esta variable determina o puede condicionar significativamente el establecimiento de la estrategia de funcionamiento y éxito empresarial, que permite lograr a través de ella el que se obtengan mejores resultados y los respectivos estándares de efectividad y eficiencia económica.

La proposición que antecede ha sido corroborada por algunos investigadores en el ámbito de la Teoría Organizacional, razón por la que el presente artículo académico analiza la cultura organizativa y su relación con la gestión empresarial, y hace énfasis sobre la efectividad, dirección estratégica y su trato como recurso intangible.

\section{MARCO CONTEXTUAL}

Si bien los orígenes de la estrategia empresarial se encuentran en el arte de hacer la guerra y la situación de juego ${ }^{1}$, es precisamente a partir de cómo evolucionan los entornos que la dirección de empresas va adaptando su manera de pensar y actuar para saber enfrentarse eficazmente a las situaciones y problemas que se presentan, logrando posiciones ventajosas en el mercado. Recordemos al respecto a Ansoff, quien hace

\footnotetext{
1 La primera -estrategia militar-, en el sentido de diseñar estratagemas, o planes de acción para poder vencer al enemigo indicándose los caminos certeros para mover y disponer las fuerzas en la contienda; mientras la segunda -estrategia del juego-, representa el conjunto complemento de instrucciones que le aconsejan al jugador la forma exacta de intervenir en todos aquellos escenarios posibles de la partida (Bueno, 1993).
}

manifiesta la denominación de Strategic Management en su artículo The concept of strategic management (Journal of Business Policy, Vol. 2, 1972), dirección estratégica que será utilizada entonces para designar un enfoque del management que permite el dirigir a la empresa exitosamente teniendo en cuenta las relaciones de esta con un entorno que es turbulento, y la necesaria y correcta adaptación de aquella a este.

Bajo esta misma perspectiva, Porter (1980), quien define a la estrategia como una forma de relacionar a la empresa con su medio ambiente y que involucra una conducta ofensiva, y/o, defensiva para lograr promover una cierta posición defendible frente a aquellas fuerzas competitivas del sector industrial en que se encuentra presente y poder conseguir, de esta manera, un rendimiento superior, hace que las empresas comiencen a preocuparse por sus desequilibrios funcionales, consecuencia de la interrelación con el entorno, problema al que se le llegó a denominar "estratégico".

Ya casi van a cumplirse cincuenta años desde que el cambio estructural y la dinamicidad del entorno, propia de los países industrializados por el acopio, tanto de factores políticos -el papel de los bloques y la guerra fría-, sociales -la sociedad postindustrial-, económicos -internacionalización del capital hacia los nuevos mercados y el protagonismo de las empresas multinacionales-, como la excesiva regulación del mercado norteamericano que llevó a las grandes empresas de este país a dirigirse a Europa en momentos de significativo crecimiento de su economía, justificaron por completo la necesidad de dar una respuesta sistémica para poder afrontar las condiciones variantes del entorno, surgiendo el paradigma de la estrategia empresarial como una de las formas de dirigir el crecimiento, o encarar la complejidad de la nueva demanda (Dalmau, 1993; Renau, 1993).

Ante tales circunstancias, se hacía necesaria la formalización de los procesos en la toma de decisiones y la coordinación e integración de las actividades de la empresa, tanto en el área funcional como de sus demás áreas. No obstante, para aquella época el aumento de la complejidad y dinamismo del entorno, con cambios más constantes, profundos y numerosos, puso en evidencia la total inconsistencia de un sistema de dirección basado solamente en políticas que fueran diseñadas ad hoc, desvinculadas unas con otras y con un horizonte temporal completamente reducido. 
De acuerdo con Sanz y Sabater (2002), tales mutaciones fueron influyendo en el pensamiento organizativo de la época hasta que se crea un nuevo enfoque que favorece la proactividad y planificación a la hora precisamente de formular las políticas, y modifican en la parte esencial a los sistemas de dirección dominantes, lo cual da lugar al conocido sistema de planificación estratégica -así, las políticas de cada área funcional deberán ser elaboradas conjuntamente con las áreas de la organización lográndose la óptima coordinación e integración entre ellas-.

Al parecer, aquel elemento tan diferenciador que justifica el surgimiento del enfoque estratégico, fue el modo de pensar y analizar del propio sistema de dirección en el sentido de ser global en relación al entorno, y corporativo, a la vez, en lo que atañe a la organización, pensamiento que en la época formaba parte del paradigma empresarial. Este precedente demostró ser bastante incompleto en cuanto, por un lado, las empresas continuaban con sus proyectos de diversificación y, por otro lado, la predictibilidad de los acontecimientos que pudiesen ocurrir en los entornos era imposible a través de la extrapolación de tendencia.

Una vez que el enfoque estratégico sufriera una sustancial modificación en su aspecto metodológico, producto de la crisis industrial de finales de los años setenta, en la que por motivo del incremento de los costos de la energía, del trabajo, del capital financiero, entre otros, los niveles productivos se desplomaron vertiginosamente -a partir de entonces, desde la perspectiva estratégica, se buscó una explicación a las etapas económicas opuestas, entre esta y aquella de expansión-, y surgió una forma de abordar los problemas estratégicos concentrada, tanto en el corto como en el largo plazo, que incorporó el enfoque estratégico en todo el proceso mismo de la dirección -desde la planificación al control, pasado por la organización, la ejecución y coordinación de actividades-; este enfoque se justificó en el sentido de dar respuesta a situaciones de gran expansión, o de un fuerte crecimiento económico y de la empresa propiamente -tratándose de productos y mercados-, como en situaciones de crisis económicas y cambios rápidos, de reducida predictibilidad que presentan un denominador común: la complejidad ${ }^{2}$ y variación del medio competitivo de la actividad empresarial.

2 Como señala Bueno (1995), los factores y situaciones que muestran la ruptura respecto a la linealidad explican el
Como se puede conjeturar de lo anterior, el problema aquí ya no consistió en coordinar aquellas actividades que a diario se presentan en la empresa, ni tampoco el modificar las políticas de un área funcional para adaptarse a los cambios del entorno. El problema fundamental una vez llegado a este punto es: ¿cómo la empresa debe relacionarse con su propio entorno?, lo que conduce sine qua non a la formulación de su estrategia de una manera formal, muy clara y específica.

En este orden de ideas, la estrategia empresarial clarificará los objetivos, misiones y visiones a ser obtenidos y los cursos de acción principales para aquello, según los recursos actuales y potenciales de la empresa a fin de lograr su inserción exitosa al entorno en el cual se asienta. Proposición que nos introduce a la teoría de los recursos y capacidades, por la cual la ventaja competitiva se la obtiene por las características, o factores diferenciadores de la empresa respecto a sus competidores, como por las capacidades y habilidades que son esenciales y que le permiten hábilmente defender y mejorar su posición competitiva en el mercado.

Ahora, por el contrario, el problema principal radica en ¿cómo la empresa crea y puede mantener una ventaja competitiva, constituyendo la base del proceso estratégico. Es decir que las empresas, en su proceso de desarrollo y de diversificación, justamente no deben ignorar a aquel recurso, ya sea tangible, o intangible que le procura una ventaja frente a otras. El tema de la cultura organizativa aparece como uno de los recursos empresariales que, en tanto sea de compleja imitabilidad, valioso, escaso y con pocas posibilidades de poder ser sustituido, permite a las empresas alcanzar, consolidar y preservar una ventaja competitiva en el tiempo (Grant, 1991; Barney, 1991-1992; Hall, 1992-1993; Peteraf, 1993; Fernández y Suárez, 1996; Rodríguez, 1997; Salas, 2000).

Se habla, entonces, de que la cultura organizativa puede facilitar -o bien dificultar-, la adaptación de la empresa al entorno, dado que cuando se plantea realizar cualquier acción que se puede considerar

concepto de "complejidad". De esta manera, las situaciones de gran expansión, o fuerte crecimiento de la empresa -tanto por productos como por mercados-, y las situaciones de crisis económica y de cambio estructural elevado que ponen en riesgo la supervivencia de la empresa, presentan un denominador común, que es definido por dicho concepto, en el medio competitivo y de la organización empresarial. 
como estratégica, debe tenerse en cuenta a aquellos valores y creencias que sustentan a la firma, ya que las relaciones entre ambos paradigmas son recíprocas. Así por ejemplo, si la cultura permite disminuir el riesgo en el proceso de fusiones y adquisiciones (Kilman et al., 1985), puede ser un factor muy decisivo para las jointventures (Schaan, 1988), ayuda a implantar la calidad total (Bernillon y Cerutti, 1989), determina las pautas para la utilización de la información (Martin, 1990), etc. Es fácil colegir en este punto, que al ser todas estas funciones importantes para la empresa-desde la diversidad de perspectivas-, la cultura organizativa será un elemento clave para la dirección estratégica.

Para reforzar la deducción de interrelación entre la estrategia y cultura organizativa, Swidler (1986, 284) como investigador señala que:

...las estrategias de acción son un producto cultural; la experiencia simbólica, los mitos y las prácticas rituales de un grupo crean modos y motivaciones, formas de experiencias organizadas y evaluación de realidades, modos de regulación de la conducta y medios para formar lazos sociales que procura recursos para construir estrategias de acción....

Sobre esta afirmación se colige que, para que las actuaciones sean correctas, se tendrá que desechar todas las concepciones erróneas acerca de cómo funciona internamente la empresa, de tal modo que los contenidos culturales estén ordenados con las expectativas venideras, puesto que de no ser así, la cultura puede generar un conjunto de motivaciones y procedimientos que posiblemente destruyan a la estrategia o, en todo caso, dificulte su implementación (Ambrosio, 1991; Gasalla, 1991; Morgan, 1993).

En el mismo orden de ideas, el hablar de los recursos que le permiten a la empresa dominar una posición competitiva frente a las demás del mercado, es referirse a los activos disponibles y controlables por ella mismo, ya sean físicos, tecnológicos, humanos, como organizativos. Algunos autores hacen notar que las configuraciones organizativas que se crean en las empresas, son cruciales para adaptarse al entorno (Wernerfelt, 1984; Teece, 1982; Montgomery y Wernerfelt, 1988; Barney, 1991), de modo que se pueda ser lo más eficientemente posible frente a nuevas realidades del management que exigen un cambio de paradigma en cuanto a cómo se gestiona la empresa, como base de todo esfuerzo estratégico.

Este argumento nos lleva a reflexionar acerca de que las empresas son diferentes entre sí, debido a los recursos y capacidades que disponen, así como por aquellas propiedades intrínsecas de los mismos. Estas juegan un rol cada vez más importante en la definición de la identidad de las empresas, ya que será necesario recurrir a su análisis para poner en marcha una determinada estrategia, más aún en base de la presencia de entornos turbulentos. Por último, las empresas deberán analizar las posibles opciones estratégicas disponibles a partir de sus recursos y capacidades, y de su valor potencial.

\section{CULTURA ORGANIZATIVA: APROXIMACIÓN CONCEPTUAL}

Es precisamente desde la antropología social, cuyo objetivo ha sido explicar la actuación de las sociedades en el tiempo, desde donde la cultura organizativa se define en relación a los criterios tanto de "observabilidad", así como de "significado" (Levine, 1990):

a.- Respecto al primer criterio, de observabilidad, se define como un modelo de conducta explícito, que en base de lograr resultados, configura un esquema integral de directrices -positivas y negativas, formales e informales-, que se encuentra registrado en la memoria colectiva y se ha establecido como verdad intersubjetiva, aceptado entre el grupo al que le servirá de guía para hacer frente a incertidumbres y ambigüedades.

b.- Relativo al segundo criterio, de significado, se define como un todo complejo que involucra conocimiento, creencia, razonamiento, costumbre, o cualquier otra capacidad, o modo adquirido por el individuo en el tiempo al formar parte de la sociedad o grupo social, elementos que al constituirse en consecuencia directa de la institucionalización de organizaciones sociales, se exteriorizan en las formas y maneras de adoptar decisiones, analizar, evaluar y entender la realidad, de interpretar las relaciones interpersonales y saber jerarquizar unos valores y normas para elegir entre alternativas en situaciones difíciles, rechazando, o aceptando otras.

Pese a que en el campo antropológico existen unas tendencias de opinión opuestas acerca de la cultura, se la considera como una estructura cognitiva de 
conocimientos y creencias que guarda relación, coherencia e isomorfismo con las actitudes y comportamientos que caracterizan a los individuos y grupos.

Puede afirmarse, entonces, que su aplicación en el ámbito de la organización empresarial ha ocasionado la aparición de determinadas posturas teóricas que, desde su punto de vista, son excluyentes y se manifiestan contrarias (Chikudate, 1991).

La confluencia de planteamientos o criterios, también se ha visto obstaculizada al abordar la cultura desde los paradigmas "racionalista" y "constructivista" de la psicología organizacional (Kopelman et al., 1990):

a.- De acuerdo con el paradigma racionalista, la cultura organizativa se identifica como una variable más de la empresa, capaz de ser gestionada para lograr determinados estándares de eficiencia económica, sobretodo, cuando se desea introducir en los códigos de la dirección la idea de implantar, de una forma adecuada, un modelo estratégico de gestión de la Calidad Total, que involucre no solo a los atributos de un producto, sino a la organización en su conjunto (Arce, 2007), y de manera específica, al desarrollo y dirección de los recursos humanos de la empresa.

b.- Mientras que para el paradigma constructivista no es que la empresa posea una cultura sino que la es en sí, sobre todo al tratarse de determinadas consistencias filosóficas que, al estar ligadas al logro de resultados, identifican plenamente a la empresa como tal.

Igualmente algunos autores, a pesar de haber definido a la cultura organizativa de una forma similar, han atendido a determinados factores que varían en el orden de la objetividad y observabilidad.

De tal manera que, mientras unos se centran en el estudio de fenómenos observables, como las actitudes y conductas, sucesos-rituales-ceremonias, lenguajes, simbologías y vestimentas (Deal y Kennedy, 1982; Martin y Siehl, 1983; Trice y Beyer, 1987), otros tienden a enfatizar aspectos que son subjetivos, como el sentido de la simbología y de los valores y creencias que son altamente valuados y compartidos por los miembros de la organización.

Ese accionar permite no solo poder descifrar el por qué de los comportamientos y actitudes y de su razón de ser, sino comprender y pronosticar con la suficiente exactitud una predisposición de resistencia, o de supeditación ante la implantación de una nueva estrategia y políticas de empresa para que pueda la cultura ser aprovechada como una herramienta clave de la administración, o gestión, y se conviertan en el eje fundamental para lograr el cambio planificado (Sathe, 1983; Smircich, 1983).

Schein (1985a), que es tal vez uno de los investigadores más reconocidos en el estudio de la cultura organizativa por ser sus trabajos referencias y base imperativa de otros, advierte que la cultura, al ser entendida como actitud y conducta, conduciría a una grave equivocación, peor aún el que se le asimile con conceptos tales como pautas que son trazadas por el equipo de trabajo, valores dogmáticos consensuados por la organización entera, filosofía de la política de la empresa, conjunto de normas que permiten la incorporación y mejor adaptación de un nuevo personal a la empresa, y/o, ambiente, o clima que se origina a través de la interrelación que se produce entre los miembros organizacionales.

Por el contrario, Schein considera a la cultura organizativa como un modelo de presunciones subyacentes básicas que, en muchas ocasiones, es inventado, o descubierto y llevado adelante por un grupo de individuos mediante su aprendizaje, cuando se pretende hacer frente a las dificultades de adaptación externas ${ }^{3}$ y de integración interna ${ }^{4}$, obteniendo resultados positivos y, por lo tanto, reteniendo significativa y muy adecuadamente la forma y manera de percibir, pensar y sentir en relación precisamente ante dichos problemas. Modo de hacer que se mantiene, transmite y se va perpetuando de generación en generación, sobre todo cuando se ha demostrado con el paso del tiempo la efectividad de la misma.

Al mismo tiempo, Schein plantea que en la tarea de especificar la cultura se debe identificar tres niveles: un primer nivel relativo a lo que denomina artefactos,

\footnotetext{
${ }^{3}$ Manera semejante de reaccionar y desenvolverse que, en base de las presiones o macroculturas del entorno, se exteriorizan en pautas, procesos y particulares estilos de razonamiento.

4 Como comportamiento único, que coadyuvado por las microculturas de los grupos existentes, va formando e imprime la personalidad de la empresa.
} 
que se refiere a los aspectos más visibles pero casi indescifrables de modo inmediato, como los diseños físicos, las capacidades tecnológicas, lenguajes y comportamientos; un segundo nivel relativo a los valores, identificados como dogmas y filosofías que predominan con absoluta concienciación respecto de lo que "debe ser", tal vez contrario a "lo que es"; el tercer nivel hace referencia a las presunciones subyacentes básicas, entendidas como aquellas intuiciones que, al ser reiteradamente válidas para solucionar un determinado problema, o vicisitudes que son anómalas de una realidad, administran instintivamente las actuaciones de los miembros de la organización constituyendo la base de la cultura a diferencia de los niveles más superficiales que son su reflejo.

Tomando en consideración estos elementos e introduciendo el rol trascendental que observa el significado de la simbología en la organización y de la cultura, las reciprocidades que se promueven entre artefactos, valores, presunciones y símbolos adquieren mayor importancia que su análisis individualizado.

En este sentido, resulta relevante tomar en consideración la propuesta de Hatch (1993), que define cuatro procesos: manifestación, que trasforma las presunciones subyacentes básicas en valores reconocibles, los que a su vez pueden, o bien mantener, o bien modificar las presunciones ya existentes; comprensión, que convierte a los valores en artefactos y viceversa, en tanto que si los artefactos provienen desde los mismos valores se tiende a reafirmarlos, mientras que si se originan de otra cultura, son muy capaces de transformar los valores hasta ahora evidentes; simbolización, instituye una relación de dependencia e influencia recíproca, sobre todo entre los artefactos -que poseen carácter objetivo- y los símbolos -de carácter consciente e inconsciente-; e interpretación, que clarifica el cómo los símbolos transforman, o son alterados por las maneras substantivas de interpretar y comprender, interviniendo en las presunciones subyacentes.

En base de lo expuesto, se afirma que de acuerdo al análisis del concepto de cultura organizativa han ido apareciendo casi simultáneamente diferentes estudios, que con sus respectivos planteamientos y matices, buscan una suerte de adaptación y encaje a escenarios específicos de la praxis.
Por otro lado, sin embargo, resulta fundamental para el propósito de clarificar el ámbito de aplicación del presente artículo, establecer un concepto que agrupe el aspecto principal de los anteriores elementos que han sido señalados:

Cultura organizativa constituye un sistema de significados que son compartidos de manera muy profunda, desde la alta dirección hasta la base operativa de una empresa, tratándose de algo sobrentendido, intangible e informal que la diferencia de las demás y, que dada su preponderancia y espíritu integrador de un universo simbólico que es externo e interno, homogeneiza en concordancia a ella a actitudes y conductas de los miembros de las organizaciones, y puede fortalecer tácitamente a todo el conglomerado de reglas, normativas, procesos, sistemas y estructuras de poder que esquematizan, especifican y condicionan a la toma de decisiones gerenciales $y$ demás políticas, prácticas, funcionamiento y actuaciones de la organización.

\section{GÉNESIS E INFLUENCIA EN LA GESTIÓN EMPRESARIAL}

Como pionera en el estudio de la cultura organizativa se puede mencionar a la Escuela de Relaciones Humanas (Menguzzato y Renau, 1991), ya que desde los años 30 ha impulsado la investigación del elemento humano en las organizaciones. Sin embargo, la aplicabilidad del término cultura a la organización empresarial es relativamente reciente, dado que apareció por casualidad en la literatura del management inglés de los años sesenta como sinónimo de "clima" (Blake y Mounton, 1964), para luego ser redescubierta a finales de los setenta y primer quinquenio de los ochenta, tras aparecer una serie y un sinnúmero de publicaciones que subrayan su influencia para el funcionamiento general de las empresas ${ }^{5}$.

\footnotetext{
${ }^{5}$ A partir precisamente del artículo de Pettigrew sobre cultura organizativa -corporativa- en la revista Administrative Science Quarterly de 1979, se despierta en la literatura del management, de comienzos de la década, un gran interés sobre el tema, y se dispone de obras como las de Deal and Kennedy y de Peters and Waterman de 1982, y varios artículos que se incluyen en una edición especial en Administrative Science Quarterly de septiembre de 1983, o en Organizational Dynamics de otoño del mismo año, o en Journal of Management Studies de mayo de 1986.
} 
Gran parte de su interés se suscitó para poder explicar el éxito del modelo de gestión empresarial japonés, y se produjeron constantes comparaciones con sus tradicionales competidores de las empresas norteamericanas, a causa de que basaban precisamente su filosofía en la combinación de un cúmulo coordinado de valores y de creencias fundamentales como sustento de un sistema gerencial de políticas, de procesos y prácticas, que a través de su disciplinado uso en la empresa, ejemplariza y robustece aquellos contenidos culturales que tienden a validarse, según así lo demuestren en función a la obtención de resultados empresariales.

A continuación se desarrolla en las siguientes secciones un análisis de la manera en que la cultura organizativa influye y condiciona el funcionamiento de las empresas. Entonces se observa:

a.- En primer lugar, un trabajo explicativo que explora la posibilidad de alcanzar la eficiencia empresarial por medio de la presencia y amalgama de algunos factores de carácter cultural que catalizan el ánimo de rendimiento de los miembros de la organización.

b.- En segundo lugar, considerando que la estrategia empresarial es un componente trascendental que da licencia a la vanguardia, estancamiento, o disolución de la empresa, se examina la relación que posee con respecto de la cultura organizativa.

c.- $Y$ en tercer lugar, haciendo una reflexión sobre las cualidades de valía y escasez, compleja imitabilidad y reducida sustituibilidad que identifican a un recurso como estratégico y fuente para disponer de una ventaja competitiva, la cultura organizativa se observa desde la óptica de estos parámetros.

Sobre la efectividad de las empresas.- Del análisis de las investigaciones que interrelacionan la cultura organizativa con la eficacia y eficiencia económica empresarial (Baker, 1980; Ouchi, 1980; Schmartz y Davis, 1981; Lorsch, 1982; Martin et al., 1983; Kilman et al., 1985; Schein, 1985a; Scholz, 1987; y otros), se llega a la conclusión de que aquella empresa que involucre un conjunto específico y apropiado de valores, creencias y demás presunciones que aumente la moral de su personal, clarifique sus conductas y canalice todo su potencial productivo hacia la consecución de los objetivos, misiones y visiones y empresariales, mejorará su funcionamiento, y se optimarán de manera substancial los resultados operativos y económicos.

Efectivamente, el considerar la importancia de que el personal de la empresa colabore y se inmiscuya cada vez más en la labor comunitaria institucional, promueve la autoestima de todos y cada uno de los diferentes estatus que conforman la empresa, dando mérito a la participación y el aporte de ideas, en la que cada individuo conoce de la responsabilidad en sus funciones y tareas, y la manera correcta de hacer las labores, proyectando eficaz y eficientemente su iniciativa a fin de perfeccionar el desempeño empresarial (Mernousch, 2002).

De acuerdo con Denison (1991), no solo es la valoración de la participación, sino el grado de compromiso compartido entre el personal de la empresa el que instituye un sentimiento de propiedad y responsabilidad que genera óptimos resultados.

Siguiendo a Deninson, la coherencia entre los principales valores, creencias y presunciones, y todo el conjunto de las políticas, procesos y prácticas hace esperar naturalmente un sistema gerencial integrado y coordinado que se suma como un elemento decisivo para conseguir una mayor eficiencia; al igual que la adaptabilidad de los contenidos culturales, que faculta a que se analice y responda de manera oportuna a las exigencias de los entornos desarrollando el potencial de reacomodo de los procesos y prácticas internas; y la misión, que al clarificar la función que demuestra la empresa y el rol de su personal, se confiere un sentido de propósito que despejan los criterios de decisión y definen las acciones más convenientes, objetivos y -en consecuencia-, los resultados en un horizonte temporal.

Al ser factible la conjunción de todos y cada uno de estos factores, se puede deducir que por ser la cultura organizativa adaptable, consistente y consecuente a la participación individual en un contexto de una misión que en su filosofía es compartida, la empresa exhibirá éxito en la consecución de sus propósitos.

A pesar de que puede existir determinada crítica ${ }^{6}$ respecto de las investigaciones que interrelacionan la

\footnotetext{
6 Por lo general, ninguna de las investigaciones que tratan el tema cultura-eficiencia económica han seguido una pauta de formular primero un conjunto de criterios que capten su teoría acerca de lo que verdadera y efectivamente hace funcionar a las organizaciones, peor aún el procurar determinada información
} 
cultura organizativa y el logro de la efectividad de las empresas, en el sentido de que son conceptualmente algo limitadas, metodológicamente defectuosas y científicamente poco convincentes (Siehl y Martin, 1990), se puede aceptar la posibilidad de que tal insuficiencia de soporte empírico, o problemática de no evidencia, no sea el resultado de la ausencia de una correlación entre la cultura organizativa y la efectividad económica, sino más bien que corresponda a una mediana dificultad metodológica el evaluar a dicha correlación, en cuanto a que en esta puedan intervenir determinados factores externos a la empresa -macroeconómicos, institucionales, o los de estructura de mercado, como el número de los competidores, cualidades intrínsecas de los productos, demanda de los consumidores, etc. (Fernández et al., 1997)-.

Del mismo modo, es probable que el rendimiento empresarial haya sido concebido de manera equívoca y que su medida debiera incorporar la estimación de factores intermediarios ${ }^{7}$.

para ratificar la validez teórica. Este descuido de los criterios de la ciencia normal, representa una fortaleza y debilidad al mismo tiempo. Sin dificultad alguna en la estructuración de la investigación, instrumentos y procedimientos, los autores de estas investigaciones resucitan a las organizaciones que estudian de una forma laudable y casi sin precedentes para aquellos que escriben sobre gerencia y organizaciones. Sin embargo, sin base para comparar organizaciones a prudente distancia, resulta un tanto difícil que el lector quede convencido por la evidencia del autor, o por sus argumentos. Una posición de defensa y no de investigación, podría oscurecer el tema y agravarlo porque en estas investigaciones exclusivamente se incluyen a organizaciones ejemplares. Ninguno de los autores estudia a las firmas que han fracasado, o que estuviesen funcionando deficientemente; además, ninguno de ellos tampoco utiliza un marco de referencia que contraste los éxitos y los fracasos. Supuestamente, si una teoría es correcta, los factores que contribuyen al éxito también deben servir para separar a las empresas exitosas de las fracasadas. Sin una prueba de la relación cultura-eficiencia económica, la ambigüedad de la respuesta posiblemente se mantenga, incluso hasta hoy.

7 A pesar de que, efectivamente, en cierta medida, se ha mantenido la idea entre estrategas, financistas y mercadotecnistas de que las grandes corporaciones son simplemente cajas negras que responden a mercados externos y fuerzas reguladoras y que solo pueden dirigirse con determinados criterios financieros, la disciplina académica del comportamiento organizacional sostiene que la diferencia entre empresas exitosas y no exitosas estriba en los valores y creencias esenciales que son la base
De igual forma, es muy probable que la cultura organizativa tenga acción directa sobre ciertos aspectos no financieros, como las actitudes y conductas del personal, la moral, motivación y satisfacción de los trabajadores, espectro definitorio de las decisiones, etc. En el sentido de que podría incidir de una forma indirecta sobre los objetivos económicos de las empresas por medio de determinadas variables como el rendimiento, innovación tecnológica, orientación al mercado, la socialización, aprendizaje y absentismo.

En definitiva, en base de lo argumentado anteriormente, se sugiere la posibilidad de que se pueda identificar aquellas cualidades específicas que favorezcan -en mayor, o en menor medida que otras-, la obtención de resultados empresariales en un horizonte temporal.

De las ideas precedentes se llega a establecer la siguiente formulación teórica que será de gran utilidad para efectos del presente artículo científico:

\begin{abstract}
Aceptando que la cultura organizativa puede incidir de una manera indirecta sobre la eficiencia de las empresas $y$, particularmente, sobre sus resultados económicos, esta acción está sujeta a la determinación y combinación de ciertos factores de naturaleza ideológica y subjetiva que exhortan a que los miembros de las organizaciones desarrollen correctamente sus funciones, encausando su esfuerzo e iniciativas al cumplimiento de aspiraciones y consecución de los objetivos y metas empresariales.
\end{abstract}

Su relación con la dirección estratégica.- En la década de los años ochenta, precisamente en el campo de la Dirección Estratégica, los argumentos de los autores giran en torno al carácter influyente que posee la cultura organizativa sobre el proceso integral estratégico de las organizaciones (Allaire y Firsirotu, 1984; Schein, 1985b;

de su organización interna. Precisamente, se pone de relieve a la cultura organizativa como una variable comportamental que generalmente tiene un impacto predecible sobre el destino de la organización. Paralelamente a esta importante premisa, Hansen y Wernerfelt (1989) y Powell (1992) consideran al "clima organizacional" como un aspecto de la organización interna empresarial que explica una parte sustancial del beneficio no atribuible a las variables que son tradicionales de lo que se entiende por estructura de mercado -calidad del producto, gustos y preferencias, capacidad adquisitiva del consumidor, posicionamiento-. 
Thévenet, 1986), entendido como una orientación fundamental de cómo la esfera directiva a través de la decisión más oportuna acerca de la actividad a ser desarrollada, o negocio que se ejerce, o se pueda ejercer, supera al cúmulo de incidencias adversas que el cambio del entorno ocasiona en el funcionamiento de aquellas.

Necesariamente a partir de estas ideas, en que la estrategia delimita el accionar de la empresa y la cultura organizativa subordina a la estrategia (Dasí, 2007b), se colige que ambas variables inciden de una manera directa en lo que la empresa "es" y "quiere ser en sí misma".

En este sentido, puede señalarse que el proceso estratégico de las organizaciones abarca determinadas etapas que mantienen necesariamente cierto ordenamiento entre sí (Claver, 1995):

a.- Una inicial, que corresponde a la formulación en la que una vez que se haya realizado el diagnóstico interno e integral de la empresa - en aspectos como su posición competitiva, financiera, productiva e investigadora, etc. -, como externo -sobre todo en lo que tiene que ver al desarrollo y configuración de la tendencia económica y tecnológica-, se clarifique el camino para que la empresa pueda alcanzar una determinada ventaja competitiva sostenible ${ }^{8}$.

\footnotetext{
${ }^{8}$ Reflexionar acerca de los motivos que empujan a las empresas a planificar una estrategia es algo prioritario, puesto que involucra que la misma empresa sea capaz de conocerse, identifique la cualidad intrínseca que la hace única y defina lo que efectivamente va a llevar a cabo para sí misma y para los grupos implicados con ella -accionistas, trabajadores, colaboradores y autoridades que la reglamentan (Besseyre Des Horts, 1989) -. Así, una vez que se conozcan los contenidos culturales a través de la examinación interna, se fijarán aquellos objetivos realistas en el contexto de un horizonte temporal (Tena-Millán, 1989). Para obtener unos determinados objetivos genéricos se irán proponiendo algunas potenciales estrategias para lograrlos mediante la búsqueda de disyuntivas concretas que luego pueden ser materia de discusión -en este tramo no puede descartarse una posible desviación cultural; de ahí que, en tanto pueda eludirse la confrontación entre la estrategia y los valores y creencias principales de la empresa, se elegirá dicha opción (Freije, 1989) - . Sin embargo, hay que anotar que esta decisión tendrá una repercusión económica que, si no es aceptada, puede ser crítica (Claver y Llopis, 1993). Será preferible que la alternativa más idónea de estrategia no obligue a transformar a los contenidos culturales, pero si no es posible, será el momento preciso de analizar y acordar si la modificación es conveniente -o no-, puesto que la empresa se va a comprometer con esta toma de decisión y el haber procedido correctamente en
}

b.- De forma secuencial, la implantación de la disposición establecida en la etapa anterior implica la utilización de recursos económicos -inversiones y gastos-, y debe existir entre ambas etapas una estrecha concordancia; no resulta suficiente el solo pensar que la implantación siga automáticamente a la formulación, puesto que el seguir lo establecido de manera prevista y dentro de los plazos, parámetros y condiciones, será de crucial importancia para que el éxito sea una realidad ${ }^{9}$.

c.- Por último, el control concurrente de la aplicación de la estrategia a través del continuo recordatorio de lo que verdaderamente se persigue y acerca de cuáles son los principales ideales, consignas, valores, creencias y presunciones que permiten dicho logro, será algo fundamental para obtener el modelo futuro que se busca de forma mediata y en el largo plazo ${ }^{10}$.

Frente a la disyuntiva de si se debe o se puede relacionar los contenidos culturales de la empresa con la estrategia o, si por el contrario, se debe rechazar toda estrategia que se encuentre en clara oposición a la cultura organizativa, considerando de manera única a aquellas estrategias que guarden consonancia con determinados valores y creencias centrales -alternativa que no deja de ser simplista, puesto que se desperdiciarían oportunidades que pueden mejorar la posición competitiva de la empresa frente a otras similares del mismo sector-, Claver argumentadamente señala que esto dependerá, en gran parte, tanto de la necesidad de una nueva implantación estratégica como del grado de fortaleza de la cultura ya existente ${ }^{11}$.

cada uno de los pasos anteriores, hará que ahora la selección sea al mismo tiempo mucho más fácil y acertada.

${ }^{9}$ De ser necesaria la modificación de los contenidos culturales en la empresa, debe producirse en la etapa de formulación, puesto que de sucederse en la de implementación, las implicaciones y posibles desavenencias culturales se pondrán de relieve -e incluso-, pueden agravarse en la media en que se profundice dicho accionar estratégico, suscitándose continuos desacuerdos por la evolución lenta que caracteriza tanto a valores como a creencias (Ambrosio, 1991; Gasalla, 1991; Morgan, 1993).

${ }^{10}$ Una manera de apoyar esta tarea es a través de los sistemas de motivación -sean estos en forma de mejoras remunerativas, simbologías de reconocimiento, ascensos, o cualquier otro tipo de estímulo que consiga reforzar los anhelados contenidos culturales en la empresa-, conocido en la literatura como estrategia cultural.

${ }_{11} \mathrm{Si}$ se proyecta introducir una nueva estrategia para apenas mejorar la posición de la empresa en el mercado, no será 
En todo caso, considerando que los diferentes efectos del cambio cultural son vivenciales, tendría justamente que evaluarse de manera concienzuda las consecuencias en el mediano y largo plazo, asumir el riesgo del cambio y estimar la viabilidad de la nueva estrategia a implantarse, ya que sólo en condiciones excepcionales -de supervivencia empresarial, proyectos interempresariales, necesidad de adaptación, reforma legal, rentabilidad frente al riesgo inminente, etc.-, se explicaría -o no-, la realización de dicho cambio.

Varias investigaciones hacen referencia al cambio cultural (Dyer, 1985; Sathe, 1985; Trice y Beyer, 1985;

deseable cambiar a la cultura de la empresa, puesto que los costos, tanto en tiempo como en dinero, serán mayores y no compensará a los beneficios potencialmente logrables. Pero si se trata de una situación muy crítica, en la que de no introducir imperativamente nuevos planteamientos que condicionan y dan soporte a la propia supervivencia de la empresa, una nueva orientación en los contenidos culturales se justifica por costosa que represente esta acción, más aún cuando las ganancias previstas sean atractivas por su cuantía. Por otro lado, taxativamente, a mayor arraigo de los valores y creencias en la empresa, mayor es la dificultad de cambio, dado que si los contenidos culturales son amplia y consistentemente asumidos por la mayor parte de los miembros la organización, el reorientar este carácter común se convierte en una dificultad muy reseñable (Scholz, 1987). La edad de la empresa es otro factor importante a ser analizado: a) en su fase de juventud, la integración cultural puede declinarse, y crearse nuevas subculturas que originarían una crisis de identidad por la pérdida de perentorios objetivos y metas, valores y creencias esenciales, ofreciéndose la oportunidad para encauzar el cambio cultural -en este sentido, no debe ignorarse también la presencia de algunos acaecimientos como la expansión y diversificación de mercados y productos, adquisiciones, fusiones, absorciones, alianzas estratégicas, integraciones vertical y horizontal, etc. -; b) en su fase de madurez, en la que se admiten graves situaciones que van desde el estancamiento operativo, el declive en los mercados, hasta la falta de predisposición para el cambio, la cultura organizativa debe actuar como promotora de la creatividad y ser considerada como una fuente de autoestima y custodia al observar los triunfos del pasado. Es aquí donde pueden producirse importantes metamorfosis que comprometen la supervivencia de la empresa, o su autodecadencia a través de hechos como la bancarrota, absorción, y/o, fusión y reorganización posterior. En este caso, los valores, las creencias y presunciones de la empresa serán así modificados, incluso en sus niveles paradigmáticos superiores, por medio de proceder al reemplazo de aquel personal considerado clave (Fernández et al., 1998) -.
Muñoz-Seca, 1989; Martín y Leal, 1989). Sin embargo, una propuesta específica respecto de los pasos que deben seguirse para la implantación de unos valores, creencias y presunciones que son fundamentales en la empresa, vienen a ser señalados por (Claver, 1995):

a.- Conocer los valores que se comparten, ya que sería inadmisible el tratar de modificar los contenidos culturales si anticipadamente no se los conoce.

b.- Precisar el motivo de la necesidad de llevar adelante un cambio cultural, al considerar la conveniencia de reorientar las actividades frente a unas circunstancias económicas, de innovación tecnológica, políticas e imposibilidades físicas y estructurales, etc.

c.- Especificar la clase y el rasgo de cultura requerida, tarea que está preestablecida por la estrategia que se pretende implantar, puesto que en algunos casos esta obligará a un mayor, o menor grado de intensidad, respecto de la modificación de unos valores, creencias y presunciones.

d.- Concienciar a la esfera directiva sobre la importancia del cambio estructural para que, a través de las actitudes y comportamientos, se irradie una nueva imagen corporativa y se ejemplarice al resto del personal colaborador en la empresa.

e.- Comunicar al personal la implantación e importancia de los nuevos valores, creencias y presunciones por razón de las alternativas existentes de comunicación -verbal, escrita, normativa, etc.-.

f.- Plasmar la modificación de valores y creencias por medio de una simbología material -diseño de edificios, calidad y novedad de los equipos y del mobiliario, tipos de vestuario, spot publicitarios de carácter escrito, gráfico y audiovisual-, e inmaterial -distinciones que son concedidas, títulos del puesto, procedimientos de acceso comunicativo del personal con la esfera directiva, lenguaje empleado, actitudes y comportamientos-.

g.- Reemplazar los programas de formación, de tal forma que el personal acate y se identifique plenamente con los contenidos culturales deseables para la empresa.

h.- Controlar de manera permanente la firmeza de las modificaciones que se han producido con el ánimo de no retroceder a la práctica de antiguos hábitos, creencias y 
demás ideologías.

Del análisis descrito en su conjunto, se llega a establecer la siguiente formulación teórica:

Resulta evidente que la cultura organizativa
puede facilitar o, muy por el contrario, impedir
o rechazar la implantación y desarrollo de una
estrategia, esta última que a más de conducir a
la empresa hacia la realización de sus objetivos
económicos, le sirve de una buena guía en su
constante búsqueda para conseguir un mejor
funcionamiento por medio de la coherencia
que debe existir entre cultura organizativa y
estrategia; se colige, por tanto, que aquella
influye en la finalidad de la actividad empresarial.

Su importancia como recurso intangible.- En la década de los años noventa la cultura organizativa es considerada como uno de los recursos y capacidad específica de las empresas que permite alcanzar, consolidar y preservar una ventaja competitiva armónica y sostenible en el tiempo, a condición de que incorpore unas características definidas, tanto de valía como de escasez, de compleja imitabilidad y con reducidas posibilidades de ser sustituida (Grant, 1991; Barney, 1991-1992; Hall, 1992-1993; Peteraf, 1993; Fenández y Suárez, 1996; Rodríguez, 1997; Salas, 2000). Por lo general, la teoría de los recursos y capacidades hace hincapié en identificar, desarrollar, proteger y desplegar los factores internos de la empresa, que por su naturaleza precisamente explican su potencial de crear valor.

A pesar a que se mantiene un vasto acuerdo en torno a la idea de que determinados valores, creencias y presunciones facilitan la implantación y desarrollo de las estrategias, existe la duda de que la cultura de la empresa sea exclusiva (Martin et al., 1983), en el sentido de poseer esta contenidos culturales que la diferencian de las demás que componen la industria y/o sector.

Sin embargo, como una manera de esclarecer dicha preocupación, se ha llegado a validar la proposición de quetal carácter de especificidad cultural, sea parte integral de un universo de empresas que mediante experiencias que son propias de su fundación, personalidades únicas que la conforman, acontecimientos exclusivos de su crecimiento y desarrollo, etc., se encuentren facultadas para generar una ventaja competitiva sostenida en el tiempo (Barney, 1986), lo que deja dicha suerte supeditada a la existencia de barreras a la imitación por parte de los competidores empresariales ${ }^{12}$.

De forma casi simultánea se ha argumentado que, del análisis de la influencia que muestra la cultura organizativa sobre el funcionamiento de las empresas, apenas aparecen sus sustitutos (Barley, 1983), inclusive en aquellas empresas que todavía no han identificado y afianzado los significados que están detrás de su actividad y que aún, procediendo a sustituir a algunas de sus políticas, procesos y estructuras internas en pro de una determinada ventaja competitiva, ha sido poco probable que dicha alteración de los procesos internos afecte significativamente a todas y cada una de las dimensiones sociales, como lo hace claramente la cultura de la empresa de una manera y forma amplias (Barney, 1992).

Esta deducción no implica -en absoluto-, que algunas de las empresas que disfrutan de determinadas ventajas competitivas fundamentadas en algunos

\footnotetext{
${ }^{12}$ Una barrera importante a la imitación se vuelve particularmente efectiva cuando los competidores no comprenden las competencias en las que se basa una determinada ventaja competitiva, surgiendo así la ambigüedad causal (Lippman y Rumelt, 1982). Sin embargo, para que las competencias puedan ser fuente de dicha ventaja y ambigüedad simultáneamente, Reed y DeFillippi (1990) proponen tres características que se deben cumplir: a) carácter tácito; considerando que las competencias se basan en la interpretación de una actuación concreta que genera un conocimiento específico -acumulado por la experiencia y perfeccionado mediante la práctica-, resulta incomprensible el codificar a las reglas y los protocolos que subyacen a dicha actuación; b) complejidad; de y entre las competencias de la empresa que garantiza que pocos, o incluso ningún individuo tenga la suficiente amplitud y profundidad de conocimientos para comprender las múltiples interacciones causales entre la cultura de la empresa y sus procesos, que dificulta cualquier posibilidad de imitación de los mismos; c) especificidad; en el sentido de que a pesar de que una empresa pueda identificar cuáles son los contenidos culturales que hacen a otra alcanzar el éxito, su imitabilidad resulta imposible debido a circunstancias únicas que hacen de la cultura específica y particular de la empresa -en este aspecto tampoco se debe olvidar la imposibilidad de relacionar exactamente lo que se piensa, supone, o anhela ("valores declarados") y la forma en que se comporta ("valores reales"), complicando la posibilidad de imitar una determinada cultura por la dificultad que entraña el descubrir aquellos valores y creencias esenciales que guían el comportamiento de la empresa-.
} 
especiales y específicos valores, creencias y presunciones, gozarán siempre de las mismas, puesto que una cultura organizativa, a pesar de ser valiosa, si no concuerda con las necesidades de la empresa, o es extremadamente rígida e inflexible y que dificulta la correcta adaptación de la empresa a entornos que son cada vez más turbulentos, puede verse, ciertamente, como un obstáculo para la supervivencia empresarial constituyendo así una desventaja (Thévenet, 1986; Kats y Rosenzweig, 1992).

Justamente la perspectiva de fortaleza cultural presenta unos determinados inconvenientes (Rodríguez, 1997):

a.- Inercia estructural: en la medida en que se intensifica la coherencia interna entre la estrategia, estructura, cultura y comportamiento del personal de la empresa, esta alcanza altos niveles de rentabilidad en el corto plazo, que produce en aquella una dificultad de adaptación al volverse renuente ante las permutaciones, ya que la consecución del éxito le ha llevado a incrementar su tamaño y complejidad en la configuración de su sistema interno de gestión, de modo que cualquier modificación se vuelve más difícil y costosa, inclusive cuando se hace necesario un espacio de tiempo para implantarla y sobre todo, si se refiere a ciertas modificaciones incrementales que son valuadas como insubstanciales y, por tanto, no imprescindibles para los elementos estructurales que pasan a conformar la empresa.

b.- Inercia cultural: en la medida en que la empresa atraviesa por su fase de madurez; más aún si en entornos estables la fortaleza de su cultura ha contribuido para lograr un determinado éxito, mayormente institucionalizados se hallan sus valores, creencias y presunciones, al igual que su sistema interno de gestión, de modo que la empresa se enfrenta a una difícil tarea al reinstitucionalizar sus contenidos culturales, políticas, procesos y prácticas internas para dirigir su actuación ante la presión de un entorno cambiante, sobre todo si su aprendizaje se ha producido en base de experiencias y expectativas compartidas sobre las formas y modos más eficientes de hacer las cosas demostrando indiferencia para querer adecuarse y aprovechar las oportunidades del entorno, limitando la capacidad de adaptación estratégica ${ }^{13}$.

${ }^{13}$ De acuerdo con el enfoque ecológico de las organizaciones, que permite que se identifique aquellas empresas que se encuentran altamente posicionadas en el mercado en función
Aunque habitualmente se puede identificar a la fortaleza de la cultura organizativa como una variable esencial por la cual se logran significativos niveles de productividad y rentabilidad, la unificación de esfuerzos hacia los objetivos, misiones y visiones comunes intensifica sobremanera la cooperación (Deal y Kennedy, 1982) y los dogmas que se instituyen encauzan al comportamiento del personal de la empresa substituyendo ambos -en gran parte-, a los sistemas formales por otros que naturalmente simplifican y no obstaculizan a la toma de decisiones (Camerer y Vepsalainen, 1988) ${ }^{14}$, cumplen de una manera efectiva el rol de especificar y explicitar las actuaciones esperadas, lo cual permite colegir, que una cultura organizativa fuerte limita sobremanera el potencial de aprendizaje y adaptación exitosa de la empresa.

de ciertos elementos intrínsecos coadyuvantes del éxito, Miller (1994) acoge la teoría del darwinismo interno, en el sentido de que en el interior de las empresas se van seleccionando o reforzando unos determinados valores, creencias y presunciones que se asocian al logro de significativas rentabilidades en rechazo de otros, calificados como infructuosos o poco importantes de manera que, con el tiempo, la cultura de la empresa se ajusta cada vez más a objetivos y metas limitados y dominantes, lo que puede encasillar a la esfera directiva en una única y exclusiva forma de ver las cosas. Un análisis complementario, Lorsch (1986) indica que si la esfera directiva de la organización comparte unos mismos valores, creencias y presunciones, observan a los acontecimientos a través de un único punto de vista, sin entender la importancia de los cambios en entornos inestables por estar cegados por contenidos culturales que ellos mismos los mantienen fuertemente arraigados. Pero aún cuando esta miopía estratégica pueda ser superada, reconociendo lo principal del cambio y el aprovechamiento de las oportunidades externas, los directivos tienden a responder a los sucesos cambiantes de acuerdo a su propia cultura, puesto que los valores, creencias y presunciones que comparten les ha servido como guía efectiva en el pasado y les sigue sirviendo y seguirá sirviendo ciertamente, como un patrón referencial para la actuación ante los acontecimientos que son nuevos.

14 En referencia precisamente a las creencias que se comparten como algo sustancial que logra la denominada eficiencia horizontal, ya que el personal de la empresa coordina automáticamente su actividad sin necesidad de una comunicación. De la misma manera se alcanzaría la eficiencia vertical, dado que al haber entendimiento entre los supervisores y los subordinados se cumple con el espíritu empresarial que reduce los costos en ambos casos. 
Efectivamente, esta conclusión puede ser una observación importante a la teoría de los recursos y capacidades que subraya la importancia de concentrar todas aquellas energías necesarias para lograr una competencia distintiva que asegure los resultados empresariales para el mediano y largo plazo.

No obstante, si la búsqueda de dicha ventaja competitiva sostenida involucra un proceso de aprendizaje selectivo, dicho accionar puede ser la causa del naufragio empresarial, si las condiciones del entorno se modifican de forma tal que la empresa no las pueda anticipar, motivo suficiente para mantener el óptimo equilibrio entre la explotación de las competencias y los paradigmas existentes con el aparecimiento de nuevas y eficaces alternativas, que puede convertirse en la clave fundamental para la supervivencia y prosperidad de las empresas, dado que en los casos de que no sea posible anticipar o prevenir los cambios sustanciales del entorno, la empresa puede encontrarse con ciertos contenidos culturales que resultan obsoletos a la hora de hacer frente a las diversas situaciones que se presentan en la actualidad (Rodríguez, 1997).

A consecuencia de lo que se ha analizado, se llega a enunciar la siguiente formulación teórica:

Por poseer cualidades específicas de valía y escasez, de imitabilidad imperfecta $y$ de insustituibilidad, la cultura organizativa constituye un activo intangible de las empresas que coadyuva a la consecución de ventajas competitivas armónicas y sostenidas, a condición de que su flexibilidad permita el aprendizaje y adaptación estratégica de las empresas a entornos competitivos que son cada vez más turbulentos.

\section{REFERENCIAS BIBLIOGRÁFICAS}

Allaire, Y.; Firsirotu, M. (1984). "Theories of Organizational Cultures”, Organizational Studies, 5 (3), pp. 193-226.

Ambrosio, E. (1991). "La cultura como base de la estrategia empresarial", Alta Dirección, 158, pp. 295-306.

Ansoff (1972). "The concept of strategic management", Journal of Business Policy, 2

Arce, B. (2007). "Las culturas organizativas y de calidad como elementos básicos en la transformación de las empresas rurales hacia la competitividad", Entelequia:
Revista Interdisciplinar, 5, pp. 143-150.

Baker, E. (1980). "Managing organizational culture", Management Review, 69 (7), pp. 8-13.

Balbastre, F.; Canet, Mª.; Fernández, R.; Redondo, A.; Revuelto, L. (1998). Organización y métodos de trabajo: dirección de la producción y recursos humanos, Editorial Civitas S.A., Madrid.

Barley, S. (1983). "Semiotics and the study of occupational and organizational cultures", Administrative Science Quarterly, 28 (3), pp. 393-414.

Barney, J. (1986). "Organizational culture: can it be a source of sustained competitive advantage?" Academy of Management Review, 11 (3), pp. 656665.

Barney, J. (1991). "Firm resources and sustained competitive advantage", Journal of Management, 17 (1), pp. 99-120.

Barney, J. (1992). "Integrating organizational behavior and strategy formulation research: a resource based analysis", Advances in Strategic Management, 8, pp. 39-61.

Bernillan, A.; Cerutti, O. (1989). "Implantar y gestionar la calidad total", Ediciones Gestión 2000 S.A., Barcelona.

Besseyre Des Horts, C. (1989). Gestión Estratégica de los Recursos Humanos, Ediciones Deusto S.A., Bilbao.

Blake, R.; Mounton, J. (1964). The management grid, Houston, TX: Gulf. En Hofstede, G. et al. (1990): Ob. Cit., pp. 286-316.

Bueno, E. (1993). "Fundamentos teóricos de la dirección estratégica", Publicaciones de la Real Sociedad Económica de Amigos del País, Valencia, pp. 139146.

Bueno, E. (1995). "La estrategia de la empresa: treinta años de evolución teórica". En R. Fernández (1995). Dirección de empresas de los noventa: homenaje al profesor Marcial-Jesús López Moreno, Ed. Civitas, pp. 29-49.

Camerer, C.; Vepsalainen, A. (1988). "The economic efficiency of corporate culture", Strategic Management Journal, 9, pp. 115-126.

Chikudate, N. (1991). "Cross-cultural analysis of cognitive systems in organizations: a comparision between Japonese and American organizations", Management International Review, 31 (3), pp. 219231.

Claver, E. (1995). La cultura empresarial como elemento cualitativo en la dirección estratégica de los años noventa. En R. Fernández (1995): Ob. Cit., pp. 217- 
238

Claver, E.; Llopis, J. (1993). "Cultura Industrial y cambio tecnológico”, III Congreso Nacional de Economía, Diciembre, La Coruña.

Dalmau, J. (1993). "Fundamentos teóricos de la dirección estratégica", Publicaciones de la Real Sociedad Económica de Amigos del País, Valencia, pp. 147-153.

Dasí, S. (2007b). "La cultura organizativa y las estrategias de la empresa", Alta Dirección, 42, pp. 57-66.

Deal, T.; Kennedy, A. (1982). Corporate cultures: the rites and rituals of corporate life, Reading MA: AddisonWesley. Existe una traducción al castellano, Deal, T. y Kennedy, A. (1985): Culturas corporativas: ritos y rituales de la vida organizacional, Fondo Educativo Interamericano, México.

Denison, D. (1991). Cultura corporativa y productividad organizacional. En R. Fernández (2003). "Evaluación del clima organizativo", Universidad de Valencia, Documento por publicar.

Dyer, W. (1985). The cycle of cultural evolution in organizations. En Kilmann, R. et al. (1985): Ob. Cit., pp. 200-229.

Fernández, E.; Montes, J.; Vázquez, C. (1997). La competitividad de la empresa: un enfoque basado en la teoría de los recursos, Universidad de Oviedo, Servicio de Publicaciones.

Fernández, R. \& Asociados (1998). La cultura y la empresa. En Balbastre et al. (1998): Ob. Cit., pp. 131-177.

Fernández, R. (1995). Dirección de empresas de los noventa: homenaje al profesor Marcial-Jesús López Moreno, Civitas S.A., Madrid.

Fernández, R. (2003). "Auditoría de la comunicación en la organización”, Universidad de Valencia, Documento por publicar.

Fernández, Z.; Suárez, I. (1996). "La estrategia de la empresa desde una perspectiva basada en los recursos", Revista Europea de Dirección y Economía de la Empresa, 5 (3), pp. 73-92.

Freije, A. (1989). Estrategias y políticas de empresa, Ediciones Deusto S.A., Bilbao.

Gasalla, J. (1991). "Nueva cultura organizacional y estrategia empresarial", Economía Industrial, 277, enero-febrero, pp. 217-229.

Grant, R. (1991). "The resource-based theory of competitive advantage: implications for strategy formulation", California Management Review, 33 (3), pp. 114-135.

Hall, R. (1992). "The strategic analysis of intangible resources", Strategic Management Journal, 13 (2), pp 145-148.

Hall, R. (1993). "A framework linking intangible resources and capabilities to sustainable competitive advantage", Strategic Management Journal, 14 (8), pp 607-618.

Hansen, G. Wenerfelt, B. (1989). "Determinants of firm performance: the relative importance of economic and organizational factors", Strategic Management Journal, 10 (9), pp. 399-411.

Hatch, M. (1993). "The dynamics of organizational culture", Academy of Management Review, 18 (4), pp. 657-693.

Hofstede, G.; Neuijen, B.; Daval, D.; Sanders, G. (1990). "Measuring organizational cultures: a qualitative and quantitative study across twenty cases", Administrative Science Quarterly, 35 (6), pp. 286316.

Kast, F.; Rosenzweig, J. (1992). Administración en las organizaciones: enfoques de sistemas y de contingencias, McGraw-Hill (Eds.), London.

Kilman, R.; Saxton, M.; Serpa, R. \& Associates (1985). Gaining control of the corporate culture, JosseyBass, San Francisco.

Kopelman, R.; Brief, A.; Guzzo, R. (1990). The role of climate and culture in productivity. En Schneider, B. (1990): Obv. Cit.: pp.282-318.

Levine, D. (1990). "Participation, productivity and the firms environment", California Management Review, 32 (4), pp. 86-100.

Lippman, S.; Rumelt, R. (1982). "Uncertain imitability: an analysis of interfirm differences in efficiency under competition", The Bell Journal of Economics, 13 (2), pp. 418-438.

Lorsch, J. (1982). "Managing culture: the invisible barrier to strategic change", California Management Review, 28 (2), pp. 95-109.

Lorsch, J. (1986). "Managing culture: the invisible barrier to strategic change", California Management Review, 28 (2), pp. 95-109.

Martín, F.; Leal, A. (1989). "¿Cómo cambiar la cultura de la organización?”, Alta Dirección, 147, pp. 363-373.

Martin, J.; Feldman, M.; Hatch, M.; Sitkin, S. (1983). "The uniqueness paradox in organizational stories", Administrative Science Quarterly, 28, pp. 438-453.

Martin, J.; Feldman, M.; Hatch, M.; Sitkin, S. (1983). "The uniqueness paradox in organizational stories", Administrative Science Quarterly, 28, pp. 438-453.

Martin, J.; Siehl, C. (1983). "Organizational culture and counterculture: an uneasy symbiosis", Organizational 
Dynamics, 12 (2), pp. 52-64.

Martin, Q. (1990). "Sistemas de información: aplicación a la empresa", ESIC Market, 70, pp. 127-142.

Menguzzato, M.; Renau, J. (1991). La dirección estratégica de la empresa: un enfoque innovador del management, Ariel (Eds.), Barcelona.

Mernousch, B. (2002). Corporate culture and financial performance: a study of corporate culture and its relationship to the financial performance of an organization, A Dissertation: Doctor Business Administration with Specialty in International Management., Nova Southeastem University.

Miller, D. (1994). "What happens alter success: the perils of excellence", Journal of Management Studies, 31 (3), pp. 325-358.

Montgomery, C.; Wernerfelt, B. (1988). "Diversification picardian rents and tobin' $s$ q", Rand Journal of Economics, 19, pp. 623-632.

MORGAN, M. (1993). "How corporate culture drives strategy", Long Range Planning, 26 (2), pp. 110-118.

Muños-Seca, B. (1989). "El cambio de cultura: diagnóstico e implementación”, Alta Dirección, 143, pp. 1-7.

Ouchi, W. (1980). "Markets, bureaucracies and clans", Administrative Science Quarterly, 25 (1), pp. 129141.

Peteraf, M. (1993). "The cornerstone of competitive advantage: a resource-based view", Strategic Management Journal, 14 (3), pp 179-191.

Peters, T.; Waterman, R. (1982). In search of excellence: lessons from America's best run companies, Harper \& Row, New York. Existe una traducción al castellano, Peters, T. y Waterman, R. (1984): En busca de la excelencia, Plaza y Janés, Barcelona.

Pettigrew, A. (1979). "On studying organizational cultures", Administrative Science Quarterly, 24 (4), pp. 570-581.

Porter, M. (1980). Competitive strategy: techniques of analyzing industries and competitors, Free Press, New York.

Powell, T. (1992). "Organizational alignment as competitive advantage", Strategic of Management Journal, 13 (2), pp. 119-134.

Reed, R.; Defillippi, R. (1990). "Causal ambiguity, barriers to imitation and sustainable competitive advantage", Academy of Management Review, 15 (1), pp. 88102.

Renau Piqueras, J. (1993). "Fundamentos teóricos de la dirección estratégica", Publicaciones de la Real Sociedad Económica de Amigos del País, Valencia, pp. 155-159.

Rodríguez, J. (1997). "La cultura organizativa como recurso competitivo: debilidades de las culturas fuertes", Septiembre, Comunicación Presentada al VII Congreso Nacional de ACEDE.

Salas, V. (2000). "La cultura en las organizaciones", Cuadernos de Economía y Dirección de la Empresa, 7, Septiembre-Diciembre, 7, pp. 341-364.

Sanz, R.; SABATER R. (2002). "Fundamentos de la dirección estratégica de recursos humanos: evolución del pensamiento en estrategia", Revista de Dirección, Organización y Administración de Empresas, 27, pp. 68-77.

Sathe, V. (1983). "Implications of corporate culture: a manager's guide to action", Organizational Dynamics, 12 (3), pp. 5-23.

Sathe, V. (1985). Culture and related corporate realities. Homewood IL: Irwin.

Schaan, J. (1988). "Cómo mejorar la probabilidad del éxito de las empresas conjuntas", Información Comercial Española, 622, octubre, pp. 33-44.

Schein, E. (1985a). Organizational culture and leadership: a dynamic view, Jossey-Bass, San Francisco. Existe una traducción al castellano, Schein, E. (1988): La cultura empresarial y el liderazgo: una visión dinámica, Plaza y Janés, Barcelona.

Schein, E. (1985b). How culture forms, develops and changes. En Kilmann, R. et al. (1985): Ob. Cit., pp. $17-43$.

Schneider, B. (1990). Organizational climate and culture, Edited by Schneider B., Jossey-Bass, San Francisco.

Scholz, C. (1987). "Corporate culture and strategy: the problem of strategic fit”, Long Range Planning, 20/4, 104, pp. 78-87.

Schwartz, H.; Davis, S. (1981). "Matching corporate culture and business strategy", Organizational Dynamics, 10 (1), pp. 30-48.

Siehl, C.; MARTIN, J. (1990). Organizational culture: a Key to financial performance? En Schneider, B. (1990): Obv. Cit., pp. 241-282.

Smircich, L. (1983). "Concepts of culture and organizational analysis", Administrative Science Quarterly, 28 (1), pp. 339-358.

Swidler, A. (1986). "culture in action: symbols and strategies", American Sociological Review, 51, april, Stanford, pp. 273-286.

Teece, D. (1982). "Towards an economic theory of the multiproducto firm”, Journal of Economic Behavior and Organization, 3, pp. 39-63.

Tena-Millán, J. (1989). "Estrategia, cultura y cambio 
organizativo", Alta Dirección, 145, pp. 55-68.

Thevenet, M. (1986). Audit de la culture dentreprise, Les Ėditions D’Organisations. Existe una traducción al castellano, Thévenet, M. (1992): Auditoria de la cultura empresarial, Díaz de Santos S.A., Madrid.

Trice, H.; Beyer, J. (1985). Using six organizational rites to change culture. En Kilmann, R. et al. (1985): Obv. Cit., pp. 370-399.

Trice, H.; Beyer, J. (1987). "How an organization's reveal its culture", Organizational Dynamics, 15 (4), pp. 5-24.

Wernerfelt, B. (1984). "A resource-based view of the firm", Strategic Management Journal, 5, pp. 171180. 